\title{
An Improved Coal and Gas Outburst Prediction Algorithm Based on BP Neural Network
}

\author{
Li Cheng ${ }^{*}$, Liu Yan-ju ${ }^{2}$ and Zhang Hong-lie ${ }^{1}$ \\ ${ }^{1}$ College of Computer and Control Engineering, Qiqihar University, Qiqihar, \\ Heilongjiang, 161006, P. R. China \\ ${ }^{2}$ Computer Center, Qiqihar University, Qiqihar, Heilongjiang, 161006, P. R. China \\ *Lcrb406@163.com
}

\begin{abstract}
The coal and gas outburst is one of complex geological disasters and its prediction is influenced by a multiple of factors, such as coal gas, ground stress, physical and mechanical properties, and complex non-linear system, which cause the low prediction accuracy. It is a favorable scheme to use the nonlinear BP neural network for the prediction algorithm design. But, the traditional BP neural network algorithm has some defects, such as the slow convergence speed and falling into the local minimum value easily. In order to remedy the defects and improve the prediction accuracy of the coal and gas outburst effectively, the improved BP neural network prediction algorithm of the coal and gas outburst is put forward in this paper. The additional momentum is adopted to adjust the network weight and to speed up the network convergence speed, and then the speed of network learning is adjusted self-adaptively and the number of iterations is reduced. Finally, the simulation of prediction of the coal and gas outburst in mine is carried out. Compared with the traditional BP neural network, the improved algorithm shows its superiorities and provides the basis for the accurate prediction of coal mine disasters.
\end{abstract}

Keywords: prediction algorithm; coal and gas outburst; neural network; additional momentum; convergence speed

\section{Introduction}

The coal and gas outburst that occurs in coal mine is one of complex geological disasters, and its high-speed gas flow can destroy the ventilation system, destroy the roadway equipment, cause the worker's asphyxia, and raise gas burning and explosion, which have brought about great harm to people's life and property and have restricted the mine production and the economic benefit improvement seriously [1]. In recent years, with the increase of depth and strength of mine mining, the coal and gas outburst accident has been increasingly surged [2]. It has played a positive role in guiding the coal mine safety production to carry out the effective coal and gas outburst prediction. The cause for the coal and gas outburst is the combined action of multiple factors from the physical properties of ground stress, gas and coal structure, so how to correctly predict the coal and gas outburst has become the complicated problem.

There exist a multiple of the coal and gas outburst prediction methods at home and abroad, which can be roughly classified as follows [3]. The prediction method based on empirical knowledge focuses on expert knowledge and experience with great subjectivity and low accuracy [4-6]. The prediction method based on mathematical model mainly depends on the rationality of the model index selection, completeness of the model, etc. Because the various influence factors for the coal and gas outburst are in a complex nonlinear relationship, it is difficult for the data model to reflect the 
complex mapping relationship accurately, which leads to the unsatisfactory prediction result $[7,8]$. And the prediction method based on the theory of the black box lays emphasis on certain input conditions to obtain the correct prediction, and in the prediction, it is unnecessary to know about the transformation relationship between input and output, the prediction result is less influenced by man's subjective [9-11]. PB neural network algorithm based on the theory of the black box shows the commendable nonlinear approximation capability, has a unique advantage in the information mapping, and captures the related laws between the influence factor existing in the coal and gas outburst data and the outburst accident, so it is widely used in the coal and gas outburst prediction. In this paper, the traditional PB algorithm of the coal and gas outburst prediction is researched and analyzed and the defects in the algorithm, and the improved BP neural network prediction algorithm of the coal and gas outburst is put forward which provides the higher prediction accuracy.

The remainder of the paper is organized as follows. The prediction theory is reviewed in Section 2. Then, the traditional prediction algorithm is analyzed and the improved prediction algorithm is presented in Section 3. In Section 4, simulation experiment results on contrastive data from both the algorithm of this paper and the traditional algorithm are demonstrated. Finally, conclusions are given with the importance and the practical value and some future work of the improved algorithm.

\section{Prediction Theory}

As one of complicated geological disasters happened in the coal mine, the coal and gas outburst is related closely with various influence factors, including geological structure, structure and thickness of coal layer, rigidity coefficient, coal and gas content, pressure and gas diffusion velocity, structure and mechanical properties of surrounding rock, original stress distribution and additional stress, concentration of coal pillar mining layer, hydrogeological conditions and so on. And there is a highly complex nonlinear relation between the coal and gas outburst and these factors, whose function relation is as follows:

$$
f(x)=f\left(x_{1}, x_{2}, \ldots, x_{n}\right)
$$

In which, the function $f(x)$ represents the degree of danger of the coal and gas outburst, and $x_{i}$ represents the various influence factors. Due to the complex nonlinear relationship between the various influence factors and the danger of the coal and gas outburst, the mathematical modeling is difficult to be effectively constructed by using the traditional prediction method, which brings out the prediction result with lower accuracy.

BP neural network can realize arbitrary complex nonlinear mapping, can build the complex mapping between the various influence factors and the coal and gas outburst accident, can effectively reduce the interference of man-made factors, can truly depict the nonlinear relation between input and output factors, and can realize the correct prediction of the coal and gas outburst[12-14].

\section{BP Prediction Algorithm}

\subsection{Traditional BP Algorithm}

BP network is a kind of feed-forward neural network based on gradient descent algorithm. It has been proved by theory that the feed-forward network with only a hidden layer is a generic function approximator [15]. In this paper, the three-layer BP network is adopted, which is composed of input layer, hidden layer and output layer. 
There are no connections between neuron nodes at the same layer, and there are interconnection between neuron nodes of each layer [16]. The learning process is as follows:

Step 1. The unit numbers of input layer, hidden layer and output layer are expressed as $N, L$ and $M$ respectively (values for $8,9,2$ ), the network input vector is $X=\left[x_{0}, x_{1}, \ldots, x_{6}\right]$, the output vector of hidden layer is $H=\left[h_{0}, h_{1}, \ldots, h_{7}\right]$, the actual output vector of network is $Y=\left[y_{0}, y_{1}\right]$, the target output vector for each mode of the training group is $D=\left[d_{0}, d_{1}\right]$, the weight from the neurons node $i$ of input layer to the neuron node $j$ of hidden layer is $V_{i j}$, and the weight from neuron nodes $j$ of hidden layer to the neuron node $k$ of output layer is $W_{i k}$. In addition, the threshold of the output neuron and that of the hidden neuron are $\theta_{k}$ and $\phi_{j}$ respectively.

Set

$$
f(a)=\frac{1}{1+e^{-a}}
$$

Then, the neuron node output of hidden layer is got as followed:

$$
h_{j}=f\left(\beta_{j}\right)=f\left(\sum_{i=0}^{6} V_{i j} x_{i}-\phi_{j}\right)
$$

By the same token, the neuron node output of output layer is got as followed:

$$
y_{k}=f\left(a_{k}\right)=f\left(\sum_{i=0}^{7} W_{i j} h_{i}-\theta_{k}\right)
$$

Step 2. The output neuron $y_{k}$ is compared with the target vector $d_{k}$ to calculate $\mathrm{M}$ output error:

$$
\begin{aligned}
& \delta_{k}=\left(d_{k}-y_{k}\right) y_{k}\left(1-y_{k}\right) \\
& \delta_{k}^{*}=h_{j}\left(1-h_{j}\right) \sum_{k=0}^{M-1} \delta_{k} W_{j k}
\end{aligned}
$$

Step 3. In turn, the adjustment quantity of each weight is calculated as followed:

$$
\begin{gathered}
\Delta W_{j k}(n)=\eta \delta_{k} h_{j} \\
\Delta V_{i j}(n)=\eta \delta_{j}{ }_{j}^{*} x_{i}
\end{gathered}
$$

In which, $\eta$ is the learning rate, and its value ranges from 0 to 1 .

Step 4. The adjustment of the network weight is performed according to the following formula:

$$
\begin{gathered}
W_{j k}(n+1)=W_{j k}(n)+\Delta W_{j k}(n)+\mu \Delta W_{j k}(n-1) \\
V_{i j}(n+1)=V_{i j}(n)+\Delta V_{i j}(n)+\mu \Delta V_{i j}(n-1)
\end{gathered}
$$

Step 5. Returning to step 1, the iteration is not continued until the prediction error reaches the expected target and weights $V_{i j}$ and $W_{j k}$ achieve the stability. 


\subsection{Defects and Improvement of Traditional BP Algorithm}

In theory, the traditional BP neural network algorithm achieves the arbitrary accuracy, but in practice, it has the defects of the slow convergence speed of network, easily falling into the local minimum value, the oscillation and so on. In order to accelerate the training speed of network and improve the prediction accuracy, BP neural network algorithm is improved by using the additional momentum item and the self-adaptive learning rate in this paper.

The additional momentum item is used to adjust the weight of the network. In order to adjust the weight of the network dynamically, the value that is proportional to the previous weight adjustment is added on each weight adjustment quantity. The formula is as follows:

$$
\Delta w(k+1)=\eta \frac{\partial E}{\partial w}+m \Delta w(k)
$$

In which, $k$ is the first $m$ momentum item coefficient, and $m_{\in}(0,0.9)$. After introducing the momentum item in BP algorithm, the effect of buffer and smoothness is realized, and the convergence speed of the network is adjusted effectively.

The self-adaptive learning rate is used to correct the network weight dynamically. In order to correct the network weight dynamically, the algorithm of the self-adaptive learning rate is used to determine automatically whether the value of learning rate $\eta$ is reasonable or not. If the decreasing speed of the error is lower, the value of learning rate $\eta$ is too small, and then the value of $\eta$ can be appropriately increased in the process of learning; conversely, the value of learning rate $\eta$ is too large and too much adjustment has be made in the network, which needs to properly reduce the $\eta$ value. The adjustment formula is given as followed:

$$
\eta(k+1)= \begin{cases}l r \_ \text {inc } \times \eta(k) & E(k+1)<E(k) \\ l r_{-} \operatorname{dec} \times \eta(k) & E(k+1)>\text { err } \text { ratio } \times E(k) \\ \eta(k) & \text { Others }\end{cases}
$$

In which, $\eta$ and $k$ are the learning rate and the number of iteration respectively; $E$ $(k)$ is the error function of the first $\mathrm{k}$ iteration; $l r_{-} i n c$ and $l r_{-} d e c$ are the increasing multiply factor and the decreasing multiply factor respectively; err_ratio is the error rate.

\subsection{The Improved BP Prediction Algorithm}

The learning process of prediction model of the coal and gas outburst based on the BP neural network is as follows: First and foremost, the sample set is trained to carry out the forward calculation through the network, calculating the error between actual output and desired output. Then according to the size of the actual error, the size of connection weight and threshold among different nodes of neural network is adjusted, so as to gradually reduce the difference between the actual output and desired output. Through the repeated process of "input mode--error calculation--error back propagation--weight adjustment", the difference between actual output and desired output is made less than the setting difference. Then the network training is ended and stored in the network, and finally the danger of the coal and gas of mine is effectively predicted.

Considering the characteristics of coal mines in China, the influence factors of the coal and gas outburst can be summarized as: mining depth, coal destruction type, gas content of coal seam, gas pressure, consistence coefficient of coal, thickness of soft layer and initial velocity of gas radiation [17]. Therefore, the input layer in BP 
network consists of 7 neuron nodes, and the output layer is composed of 2 neuron nodes which are set to $\mathrm{Out}_{1}$ and $\mathrm{Out}_{2}$. When $\mathrm{Out}_{1}=1$ and $\mathrm{Out}_{2}=1$ exist, it means the high danger of outburst. When $\mathrm{Out}_{1}=1$ and $\mathrm{Out}_{2}=0$, or $\mathrm{Out}_{1}=0$ and $\mathrm{Out}_{2}=1$, it means the low danger of outburst. When $\mathrm{Out}_{1}=0$ and $\mathrm{Out}_{2}=0$, it means no danger of outburst.

After a large number of experiments, the neuron of network hidden layer is set to 8 , which adopts BP network structure with $7 \times 8 \times 2$ to predict the coal and gas outburst. The network structure is shown in Figure 1.

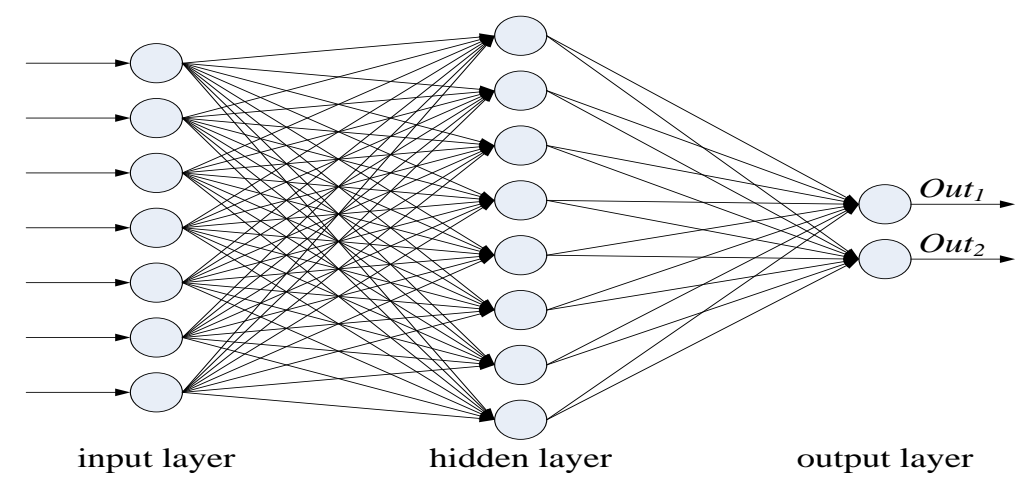

\section{Figure 1. BP Neural Network Structure of the Coal and Gas Outburst Prediction}

The prediction steps of the coal and gas outburst are shown as followed:

Step 1. For the selection of the influence factors, the influence factors of the coal and gas outburst are collected and the related factors are standardized.

Step 2. The three-layer BP neural network structure is designed. According to the related influence factors of the coal and gas and the related output, the BP neural network structure is set for $7 \times 8 \times 2$.

Step 3. The BP neural network is trained. In the training process, in order to accelerate the convergence speed of the algorithm, the additional momentum item and the self-adaptive learning rate are used to improve the BP neural network.

Step 4. The test set is used to predict the coal and gas outburst and get the corresponding safety level of coal and gas of coal mine.

\section{The Experiment and Simulation}

\subsection{The Experimental Data}

For the coal and gas outburst occurred in coal mine is one of pretty complex geological disasters, there are many influence factors. In this research, seven indicators are selected to predict the coal and gas outburst, and the original data are shown in Table 1, including eight sets of data totally.

Table 1. Original Data of Influence Factor for Coal and Gas Outburst

\begin{tabular}{|c|c|c|c|c|c|c|c|}
\hline $\begin{array}{c}\text { sample } \\
\text { no. }\end{array}$ & $\begin{array}{c}\text { mining } \\
\text { depth } \\
(\mathrm{km})\end{array}$ & $\begin{array}{c}\text { coal } \\
\text { destruction } \\
\text { type }\end{array}$ & $\begin{array}{c}\text { gas } \\
\text { content of } \\
\text { coal seam } \\
\left(\mathrm{m}^{3} \mathrm{t}^{-1}\right)\end{array}$ & $\begin{array}{c}\text { gas } \\
\text { pressure } \\
(\mathrm{MPa})\end{array}$ & $\begin{array}{c}\text { consistence } \\
\text { coefficient } \\
\text { of coal }\end{array}$ & $\begin{array}{c}\text { thickness } \\
\text { of soft } \\
\text { layer } \\
(\mathrm{m})\end{array}$ & $\begin{array}{c}\text { initial velocity } \\
\text { of gas } \\
\text { radiation } \\
\left(\mathrm{ms}^{-1}\right)\end{array}$ \\
\hline 1 & 0.620 & 3 & 10.02 & 2.76 & 0.31 & 1.20 & 19.00 \\
\hline 2 & 0.445 & 5 & 13.04 & 0.95 & 0.24 & 2.00 & 6.00 \\
\hline 3 & 0.462 & 3 & 10.36 & 1.20 & 0.16 & 1.30 & 18.00 \\
\hline
\end{tabular}




\begin{tabular}{|l|l|l|l|l|l|l|l|}
\hline 4 & 0.395 & 1 & 9.04 & 1.17 & 0.61 & 1.61 & 5.00 \\
\hline 5 & 0.745 & 3 & 9.01 & 1.25 & 0.36 & 1.41 & 8.00 \\
\hline 6 & 0.425 & 3 & 10.25 & 2.80 & 0.59 & 1.82 & 8.00 \\
\hline 7 & 0.460 & 1 & 9.50 & 2.00 & 0.48 & 1.10 & 7.00 \\
\hline 8 & 0.543 & 3 & 8.23 & 3.95 & 0.22 & 0.93 & 14.00 \\
\hline
\end{tabular}

\subsection{Experiment and Results of Prediction Performance}

Matlab7 is adopted for the experimental simulation software platform. Matlab simulation program is edited, newff( ) function is used to construct the network architecture, the init( ) function is for initialization, and $\operatorname{sim}($ ) function is for simulation. To illustrate the accuracy of the improved BP prediction algorithm, the contrastive test with the traditional BP algorithm is performed.

Table 2. Prediction Result Comparison

\begin{tabular}{|c|c|c|c|}
\hline No. & actual situation of danger & the traditional BP algorithm & the improved BP algorithm \\
\hline 1 & high & $(1,1)$ & $(1,1)$ \\
\hline 2 & low & $(1,0)$ & $(1,0)$ \\
\hline 3 & low & $(0,1)$ & $(0,1)$ \\
\hline 4 & no & $(0,0)$ & $(0,0)$ \\
\hline 5 & low & $(1,1)$ & $(1,0)$ \\
\hline 6 & low & $(0,1)$ & $(0,1)$ \\
\hline 7 & no & $(0,0)$ & $(0,0)$ \\
\hline 8 & low & $(1,1)$ & $(1,1)$ \\
\hline
\end{tabular}

From Table 2, it can be seen that the prediction performance of the traditional BP neural network is obviously worse than that of the improved BP neural network, and prediction results of the 5th bleak look-out from the traditional BP neural network do not tally with the actual situation, while the improved BP neural network achieves the correct prediction for all mines and the actual look-out danger level of the coal and gas outburst is exactly the same with higher accuracy.

\subsection{Experiment and Results of Convergence Speed}

To illustrate the superiority of the improved BP algorithm in the convergence speed, the traditional BP algorithm is compared in the experiment. It is observed that in the training, with the constant adjustment of the additional momentum item and the value of learning rate, and in the same error accuracy request, the convergence speed of the improved BP neural network model is much faster than that of the conventional BP neural network model. The comparison results are shown in Table 3 : 
Table 3. Convergence Speed Comparison

\begin{tabular}{|c|c|c|c|c|}
\hline \multirow{2}{*}{ accuracy } & \multicolumn{2}{|c|}{ the traditional BP algorithm } & \multicolumn{2}{c|}{ the improved BP algorithm } \\
\cline { 2 - 5 } & iteration time & calculation time $(s)$ & iteration time & calculation time $(s)$ \\
\hline 0.0005 & 218 & 11 & 273 & 15 \\
\hline 0.0003 & 332 & 17 & 437 & 25 \\
\hline 0.0001 & 503 & 26 & 678 & 39 \\
\hline
\end{tabular}

\section{Conclusions}

In this paper, after researching and analyzing the traditional BP neural network algorithm, the improved algorithm is proposed, which ensures the accuracy of the prediction effectively and improves the prediction speed greatly. The improved algorithm adopts the additional momentum item and the self-adaptive learning rate to improve BP neural network, which overcomes the slow convergence speed, the easily falling into local minimum value and the frequent oscillation existing in the traditional BP network to certain degree. It predicts the danger level of the coal and gas outburst effectively, and then the effective management measures are taken to prevent the happening of the danger and the working environment of workers are improved, so as to achieve the purpose of safety production as well as the work efficiency improvement.

\section{Acknowledgements}

This work is supported by National Natural Science Foundation of China for Youth, No.61403222, and the National Nature Science Foundation of Heilongjiang Province, China, No.F201440 and No.F201439. The authors also very gratefully acknowledge the helpful comments and suggestions of the reviewers, which have improved the presentation.

\section{References}

[1] A. Wu, J. Yao and H. Xiao, "Optimization for prediction index of coal and gas outburst base on gray associated analysis", Coal Science and Technology, vol. 33, no. 4, (2005), pp. 55-58.

[2] Y. Tian and L. Zhou, "The study on the methods for predicting coal or gas outburst based on BP neural network", Systems Engineering-Theory \& Practice, vol. 12, (2005), pp. 102-106.

[3] H. Dai, "Forecasting coal and gas outburst based on improved adaptive support vector machine", Application Research of Computers, vol. 26, no. 5, (2009), pp. 1656-1658.

[4] J. Hao, C. Yuan and D. Han, "Development of an Integrated Expert System for Regional Prediction of Coal and Gas outbursts", Journal of Liaoning Technical University (Natural Science), vol. 9, no. 3, (2000), pp. 240-243.

[5] L. Sun, R. Li and Y. Qu, "Application research of knowledge acquisition model for colliery gas porecast", Computer Engineering, vol. 35, no. 12, (2009), pp. 169-171.

[6] L. Wang, "Knowledge acquisition approach based on rough sets theory for gas forecast expert system of coal mine", Industry and Mine Automation, vol. 39, no. 3, (2013), pp. 49-52.

[7] J. Qiao, "Application of three mathematical models for marine economy forecasting in China", Journal of Guangdong Ocean University, vol. 28, no. 2, (2008), pp. 16-19.

[8] Q. T. Hu, S. N. Zhou and X. Q. Zhou, "Mechanical mechanism of coal and gas outburst process", China Coal Soc, vol. 33, no. 12, (2008), pp. 1368-1372.

[9] Y. Gao and C. Jiang, "Application of fuzzy mathematics in coal and gas outbursts prediction", Jiangxi Coal Science \& Technology, vol. 2, (2006), pp. 6-8.

[10] Y. Min Lü, D. Zhen Tang, H. Xu and S. Tao, "Productivity matching and quantitative prediction of coalbed methane wells based on BP neural network", Science China Technological Sciences, vol. 54, no. 5, (2011), pp. 1281-1286.

[11] J. Liu, H. Chang, T. Y. Hsu (X. Zuyao) and X. Ruan, "Prediction of the flow stress of high-speed steel during hot deformation using a BP artificial neural network", Journal of Materials Processing Technology, vol. 103, no. 2, (2000), pp. 200-205.

[12] J. Hao, "Application of improved BP network in prediction of coal and gas outburst", Journal of Liaoning Technical University, vol. 30, no. 2, (2004), pp. 9-11. 
[13] H. Xiao, X. He and L. Liu, "Application of modified BP neural network in predicting coal and gas outburst", China Safety Science Journal, vol. 13, no. 9, (2003), pp. 59-62.

[14] D. Guo, N. Li, D. Pei and D. Zheng, "Prediction method of coal and gas outburst using the grey theory and neural network", Journal of University of Science and Technology Beijing, vol. 29, no. 4, (2007), pp. 354-357.

[15] T. Chen, H. Chen and R. Liu, "Approximation capability in $\mathrm{C}(\mathrm{Rn})$ by multilayer feedforward networks and related problems", IEEE TransNN, vol. 6, no. 1, (1995), pp. 25 -30.

[16] H. Chu and H. Lai, "An improved back-propagation NN algorithm and its application", Computer Simulation, vol. 24, no. 4, (2007), pp. 75-77, 111.

[17] C. Li, R. Chen and H. Su, "Application of BP neural network in prediction of coal and gas outburst", Mining \& Metallurgy, vol. 19, no. 3, (2010), pp. 21-23. 